\title{
Uma Análise Bibliométrica Sobre Compras Públicas em Projetos de Inovação
}

\author{
A Bibliometric Analysis on Public Purchases in Innovation Projects
}

\author{
Luciana Maria de Oliveira Cortinhas ${ }^{1}$ \\ Sanderson César Macedo Barbalho ${ }^{1}$ \\ ${ }^{1}$ Universidade de Brasília, Brasília, DF, Brasil
}

\begin{abstract}
Resumo
O estudo objetivou realizar uma análise bibliométrica quantitativa acerca do tema Compras Públicas em Projetos de Inovação. Publicações sobre o assunto foram identificadas por meio de consultas na base de dados Scopus com limitação de período até 2017. Os resultados destacam os autores que mais publicam na área, os periódicos mais relevantes, os tipos de documentos utilizados para divulgação das pesquisas, as áreas às quais o tema está vinculado, os países mais interessados na temática, o período em que o tema apresentou maior ascensão e o posicionamento do Brasil nas pesquisas sobre esse assunto. Primeiramente foi elaborado um portfólio composto de 235 documentos, que contribuíram para os resultados apresentados no início do estudo, após, foram identificados os 40 documentos mais referenciados, entre 2007 e 2017, com autores e palavras-chave mais citados. O estudo ainda proporciona a continuação da pesquisa por meio de uma análise qualitativa do portfólio preparado.
\end{abstract}

Palavras-chaves: Projeto de Inovação. Compra Governamental. Aquisição Pública.

\begin{abstract}
The study aimed to perform a quantitative bibliometric analysis on the theme Public Procurement in Innovation Projects. Publications on the subject were identified through consultations in the Scopus database with period limitation until 2017. The results highlight the authors who publish the most in the area, the most relevant journals, the types of documents used to disseminate the research, that the theme is linked, the countries most interested in the subject, the period in which the theme presented the greatest rise and the position of Brazil in the research on this subject. Firstly, a portfolio composed of 235 documents was created, which contributed to the results presented at the beginning of the study, after which the 40 most referenced documents between 2007 and 2017 were identified, with authors and keywords most cited. The study also provides the continuation of the research through a qualitative analysis of the portfolio prepared.
\end{abstract}

Keywords: Innovation Project. Government Purchase. Public Acquisition.

Áreas Tecnológicas: Inovação. Prospecção. Desenvolvimento Bibliográfico. 


\section{Introdução}

A inovação tecnológica impulsiona o desenvolvimento econômico. As empresas, para se manterem em destaque no mercado, necessitam de ideias e de projetos de inovação cujos processos precisam ser cada vez mais dinâmicos e ágeis. Projetos dessa natureza possuem características de alta complexidade e riscos, que devem ser assumidos pelas empresas que optam pela inovação (GOELZER et al., 2014).

Um dos processos mais relevantes para a inovação, e que pode elevar os riscos relacionados com o projeto, é a compra de materiais e produtos. É possível encontrar na literatura alguns estudos de caso que tratam da gerência de aquisição de projetos diferentes em países em desenvolvimento. Um exemplo é o processo de aquisição de projetos de rodovias no Nepal. Manavazhi e Adhikari (2002) identificaram uma provável correlação entre o impacto de custo de um item e o atraso da sua data prevista de entrega. Os autores concluíram que problemas estruturais da cadeia de fornecimento, como monopólios e dificuldades de importação, são responsáveis por $79 \%$ dos atrasos nas aquisições. Também Fripong, Oluwoye e Crawford (2003), que entrevistaram, em Ghana, proprietários, consultores e contratantes de projetos de perfuração de poços, concluíram que os principais fatores que afetaram o atraso de projetos e ocasionam a elevação do custo final estão relacionados às aquisições. Eles elencaram como principais fatores: a ausência de gerência eficaz de aquisições, a entrega atrasada dos itens adquiridos e as dificuldades de importação, além de falhas na gestão de contratos e dificuldades de numerário para itens com pagamento antecipado (FRIPONG; OLUWOYE; CRAWFORD, 2003).

Os projetos de inovação em execução demandam materiais e produtos específicos que possuem características bastante peculiares e que precisam estar disponíveis no momento exato em que se fazem necessários de acordo com o estipulado no plano de trabalho aprovado. Barbalho, Richter e Rozenfeld (2008), utilizando o ferramental proposto por Tapping e Shuker (2003), realizaram propostas de melhorias no processo de aquisição de materiais e componentes importados a serem utilizados em projetos de novos produtos. Eles realizaram um estudo de caso com uma empresa brasileira que continha 350 funcionários, com sede em São Carlos/SP e mais três filiais, estando uma filial localizada no estrangeiro. Após terem sido detectados o volume de aquisições de itens para novos produtos e o processo de aquisição nacional e internacional, foram aplicados os conceitos de desenvolvimento enxuto na melhoria do processo de aquisição. No caso analisado, verificou-se que o maior gargalo se encontrava no processo de aquisição de itens importados, por esse motivo, tal processo passou a ser monitorado e houve a proposta de implementação de mudanças na estrutura organizacional do processo de aquisição, com critérios e metas previamente estabelecidos para os setores envolvidos.

Realizar o planejamento e o controle das compras de um projeto é um desafio para as empresas atuantes no desenvolvimento de novos produtos e/ou tecnologias, principalmente se utilizados os métodos de gestão tradicionais. Corrêa e Corrêa (2012) apresentam a gestão de produção e operações como um campo de estudo cujos focos ou fatores principais estão na forma como os recursos humanos são gerenciados e sua interação com as partes não humanas da organização, como a tecnologia, os sistemas, os procedimentos e as instalações. 
O Guia do Conhecimento em Gerenciamento de Projetos - Guia PMBOK (2013) elenca o gerenciamento das aquisições como uma das áreas de conhecimento necessárias para a execução de um projeto. Em relação à sua relevância para o adequado gerenciamento de um projeto, a gestão de compras está no mesmo nível de importância das gestões de riscos, recursos humanos, qualidade, tempo, custos, escopo, comunicações e integração das partes interessadas. O projeto de inovação não conseguirá progredir, mesmo que haja recursos financeiros suficientes, se o gerenciamento de aquisições e contratações não for bem executado.

Apesar da relevância dos processos de aquisição para a boa execução dos projetos de inovação, a legislação brasileira sobre o tema parece ainda estar aquém do necessário para proporcionar às instituições nacionais formas adequadas de adquirir itens ou equipamentos imprescindíveis para seus projetos, o que é agravado, em grande medida, no ambiente público.

Este estudo examina um conjunto de publicações sobre Compras Públicas e Projetos de Inovação, desenvolvendo uma análise bibliométrica de documentos selecionados de uma plataforma de base de dados, de modo a determinar o ano em que as discussões sobre o tema foram iniciadas, o período em que houve maior atenção ao assunto, os periódicos e as universidades que mais realizaram pesquisas, os autores mais alinhados com a questão, os países em que houve maior número de publicações, as áreas de afinidade ao tema, bem como os tipos de documentos produzidos. Por fim, é construído um portfólio com as publicações mais referenciadas no período de 2007 a 2017, com indicação de citações/ano, índice de impacto e palavras-chave.

\section{Metodologia}

$\mathrm{O}$ artigo se originou da percepção de uma lacuna na literatura brasileira sobre o tema, o que gerou inquietação sobre sua evolução nas academias brasileira e mundial. Essa dúvida gerou a seguinte questão de pesquisa: $\mathbf{O}$ que existe na literatura interdisciplinar sobre a temática compras governamentais em projetos de inovação? A partir dessa pergunta, foram selecionadas as publicações relacionadas a compras públicas e projetos de inovação, e, por fim, foi realizada a análise bibliométrica quantitativa e a criação de um portfólio bibliográfico acerca desse assunto.

A bibliometria consiste na aplicação de técnicas estatísticas para descrever a análise quantitativa da informação. Ficou originalmente conhecida como "bibliografia estatística", termo utilizado por Hulme no ano de 1923, sendo que a nomenclatura contemporânea "bibliometria" foi desenvolvida por Otlet, em 1934 (ARAÚJO, 2006).

A análise bibliométrica é uma técnica para o mapeamento dos principais autores, periódicos e palavras-chave sobre determinado tema (PRITCHARD, 1969). Ela examina o material bibliográfico de uma perspectiva objetiva, quantitativa, que é útil para organizar a informação em um campo temático específico (MERIGÓ; GIL-LAFUENTE; YQGER, 2015). É também uma forma de análise de publicações científicas que avalia a evolução do conhecimento de um assunto específico, a qualidade científica e a influência de obras e fontes (BOUYSSOU; MARCHANT, 2011; DAIM et al., 2006). 
A presente análise bibliométrica seguiu seis etapas: 1) definição do campo de estudo; 2) escolha do banco de dados; 3) ajuste dos critérios de busca; 4) compilação das categorias de informações bibliográficas; 5) codificação do material recuperado; e, finalmente, 6) análise da informação.

Destaca-se, oportunamente, que todos os procedimentos descritos neste trabalho foram realizados por meio de pesquisa na base Scopus, realizada pela última vez no dia 25 de junho de 2018.

\subsection{Detalhamento da Pesquisa na Base de Dados}

Escolheu-se a base científica Scopus porque é uma base de dados já consolidada em pesquisas científicas na área acadêmica. O segundo passo foi a seleção de palavras-chave.

Inicialmente como palavra-chave utilizou-se o termo "project management". A busca ocorreu, com restrição de período temporal de títulos publicados até 2017, em títulos, resumo, palavra-chave e resultou em 95.334 trabalhos. Com a finalidade de filtrar os trabalhos para melhor atender ao tema da pesquisa, utilizou-se o termo "innovative project" - que, utilizado isoladamente, demostrava 1.566 resultados - mas quando pesquisado conjuntamente com o termo anterior reduziu drasticamente o número de trabalhos para 205.

No intuito de refinar ainda mais a busca, foram aplicados separadamente os termos "public purchase" (61), "government purchase" (256), "public acquisition" (59) e "public procurement" (1.914). Em que pese essas palavras-chave terem apresentado o número de títulos elencados nos parênteses quando consultadas isoladamente, não foram obtidos resultados quando associadas aos dois termos anteriores. Por esse motivo, optou-se por alterar o padrão de busca.

Iniciando novamente a seleção de palavras-chave, escolheu-se a palavra "innovation". A busca ocorreu, também com restrição de período temporal até 2017, em títulos, resumo, palavra-chave e resultou em 330.888 trabalhos. Para filtrar tais trabalhos, foram utilizados juntamente com a palavra-chave escolhida os termos relacionados a compras governamentais em inglês, com AND "public purchase" resultou apenas um trabalho, com AND "government purchase" foi possível encontrar 10, com AND "public procurement" foram localizados 224 e com AND "public acquisition" apenas um. Utilizou-se, assim, a fórmula: ("innovation" AND "public purchase" OR "government purchase" OR "public procurement" OR "public acquisition"). Verificou-se que apenas um título se repetia nas referências bibliográficas encontradas, assim, 235 trabalhos perfazem a amostragem deste estudo.

Tendo sido definida a amostragem da pesquisa, iniciou-se a tabulação dos dados das 235 publicações, buscando identificar o ano em que as discussões sobre o tema foram iniciadas e em qual período houve maior debate, quais foram os autores que mais pesquisaram o tema, quais os periódicos, países e universidades apresentaram maiores números de publicações, quais os tipos de documento foram utilizados para divulgação dos trabalhos realizados, quais as áreas de conhecimento melhor exploraram o tema e como se deu a evolução dessas produções ao longo do tempo. 
Apresentados esses resultados foram delimitadas as 40 publicações mais referenciadas. Ainda na base Scopus, foram aplicados os seguintes procedimentos: $1^{\circ}$ ) foram selecionadas todas as 235 publicações com solicitação para acesso à visão geral de suas citações (citation overview); $2^{\circ}$ ) foi aplicado o filtro de data para 2007 a 2017 para concentrar a amostra nas publicações que foram mais citadas na última década; $3^{\circ}$ ) optou-se por excluir as autocitações (self-citations) de todos os autores; $4^{\circ}$ ) foram organizadas as publicações por ordem decrescente de citações (das com maior número para as com menor); e $5^{\circ}$ ) foram exportadas para um arquivo Excel todas as informações sobre título, autores, ano da publicação, periódico, citações por ano, citações totais do período delimitado, índice de impacto da citação e palavras-chave.

A bibliometria considera os aspectos do estudo a serem realizados incorporando a contagem de artigos, publicações, citações, ou seja, manifestações de informação estatisticamente significativas que foram armazenadas independentemente da área de conhecimento (GROOS; PRITCHARD, 1969).

A análise de citações tem sido utilizada como medida para o estudo da comunicação (ZHAO; LOGAN, 2002), permitindo a avaliação de informações científicas (FRANCK, 1999) e orientando o leitor para possíveis fontes de conhecimento. Nas últimas três décadas, a análise de citações tem sido cada vez mais utilizada para quantificar a importância de pesquisadores e pesquisas científicas e é o principal item considerado no cálculo do fator de impacto do documento (MEHO, 2007).

De acordo com De Bellis (2009), na análise de palavras-chave, assume-se que os autores escolhem termos científicos a partir de um repertório finito e codificado, usando termos diferentes quando postulam relações não triviais entre conceitos. Nesse caso, o reconhecimento da mesma relação entre palavras por diferentes autores revela algo sobre a estrutura cognitiva da área de pesquisa estudada. A utilidade do estudo de palavras-chave é citada por vários autores, tanto para analisar o conteúdo de publicações quanto para investigar tendências na área de estudo (SALTON; MCGILL, 1983; ELAM; HUBER; HURT, 1986; BARKI; RIVARDI; TALBOT, 1993).

A seguir, serão apresentados os resultados da análise da investigação quantitativa, servindo de pressuposto para síntese das informações e posteriores considerações sobre o tema.

\section{Resultados e Discussão}

Verificou-se que os estudos ligados às compras governamentais em projetos de inovação datam a partir de 1984. Por meio de uma linha do tempo, pôde-se observar que houve uma tendência de crescimento da quantidade de publicações sobre o tema, com considerável crescimento a partir do ano 2010, e o ápice de publicações se deu no ano de 2014.

A predominância do tema no ano de 2014 pode ser observada na Figura 1 (Publicações no tempo) e será novamente demonstrada na Tabela 2 (Publicações por área países no tempo), em que é possível verificar que a soma das publicações dos 18 países selecionados é maior nesse ano. 
Figura 1 - Publicações no tempo

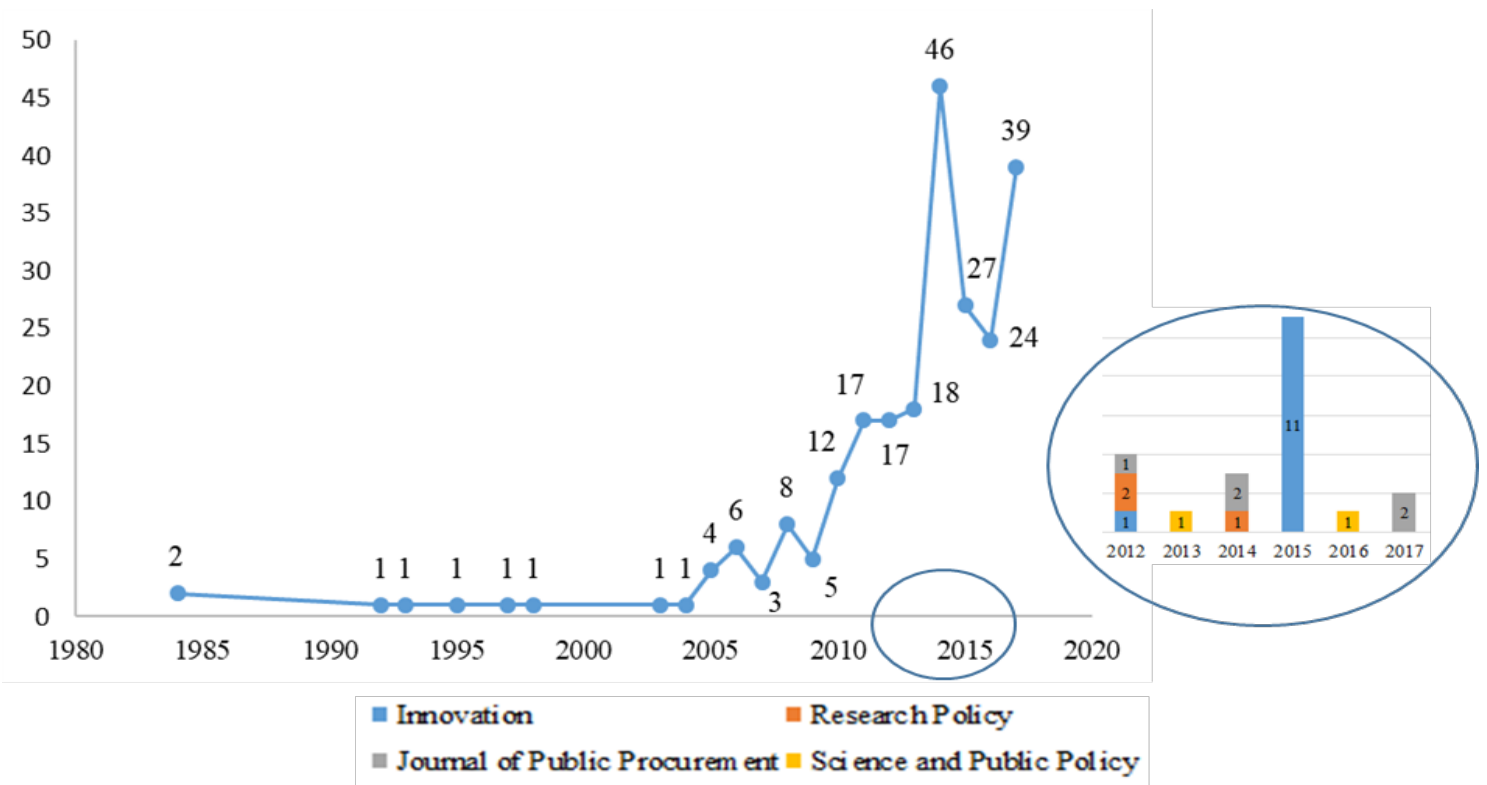

Fonte: Elaborada pelos autores deste artigo com dados da base Scopus (2018)

Da análise das publicações evidenciadas na pesquisa, no período específico de 2012 a 2017, verifica-se que a presença da temática estudada em alguns periódicos internacionais (Figura 1), como no caso da Innovation, com 12 publicações, surgindo como a revista que mais publicou sobre o tema em comparação com as demais, seguida da Journal Of Public Procurement, com cinco, Research Policy, com três publicações, e Science and Public Policy com duas.

As quatro revistas citadas têm como áreas temáticas as ciências sociais (geografia, planejamento, desenvolvimento e administração pública); negócios, gestão e contabilidade (estratégia e gestão de tecnologia e inovação); engenharia e ciência ambiental (gestão, monitoramento, política e direito).

Foi possível observar ainda o aparecimento e o crescimento de publicações sobre o tema na revista Innovation a partir do ano de 2010 com ápice em 2015. Essa revista pertence ao grupo Taylor \& Francis e foi fundada em 1798, ela conta com mais de dois séculos de história e de colaboração com a sociedade acadêmica. Ela também é uma das principais editoras do mundo, possui sede no Reino Unido, conta com mais de 6.500 funcionários e tem presença em todas as principais regiões geográficas do mundo. Publica mais de 2.500 periódicos e mais de 5.000 novos livros por ano, com uma lista de livros de mais de 120.000 títulos especializados em múltiplas áreas e em setores.

O crescimento de publicações sobre o assunto pesquisado nos periódicos da revista Innovation denota que a temática vem sendo valorizada nos últimos anos por cientistas, pesquisadores, acadêmicos e profissionais de diversas regiões e diferentes áreas de atuação. Na Tabela 1, nota-se que, dos 235 documentos analisados, alguns artigos estão classificados em mais de uma área do conhecimento e, por isso, podem ser classificados como interdisciplinares. 
Tabela 1 - Publicações por área do conhecimento

\begin{tabular}{ccc} 
Área do ConHEcimento & Publicações & PerCeNtuAL \\
Business, Management and Accounting & 105 & $44,68 \%$ \\
Social Sciences & 93 & $39,57 \%$ \\
Economics, Econometrics and Finance & 51 & $21,70 \%$ \\
Engineering & 46 & $19,57 \%$ \\
Environmental Science & 31 & $13,19 \%$ \\
Computer Science & 24 & $10,21 \%$ \\
Decision Sciences & 18 & $7,65 \%$ \\
Energy & 9 & $3,82 \%$ \\
Mathematics & 7 & $2,97 \%$ \\
Medicine & 6 & $2,55 \%$ \\
Arts and Humanities & $2,55 \%$ \\
Multidisciplinary & 6 & $1,70 \%$ \\
Psychology & 4 & $1,70 \%$ \\
Chemical Engineering & 4 & $1,27 \%$ \\
Materials Science & 3 & $1,27 \%$ \\
Physics and Astronomy & 3 & $1,27 \%$ \\
Biochemistry, Genetics and Molecular Biology & 3 & $0,85 \%$ \\
\hline \hline
\end{tabular}

Fonte: Elaborada pelos autores deste artigo com dados da base Scopus (2018)

Observando a Tabela 1, pode-se destacar as áreas de Negócios, Gestão e Contabilidade com 44,68\% do total de publicações, seguidas pelas áreas de Ciências Sociais com 39,57\% e Economia, Econometria e Finanças com 21,70\%.

Outra análise realizada é referente ao ranking dos autores que mais publicaram sobre o tema, até 2017 (Figura 2). Nesse sentido, Edler, com 10 publicações, foi o autor que mais publicou; Kalvet, Rolfstam e Uyarra possuem nove; Lember possui oito; Georghiou possui sete; e Kattel e Yeow possuem seis. Dos Brasileiros: Furtado e Ribeiro possuem duas; Abramo, Bismarchi, Cantu e Dos Santos possuem uma. 
Figura 2 - Publicações por autores

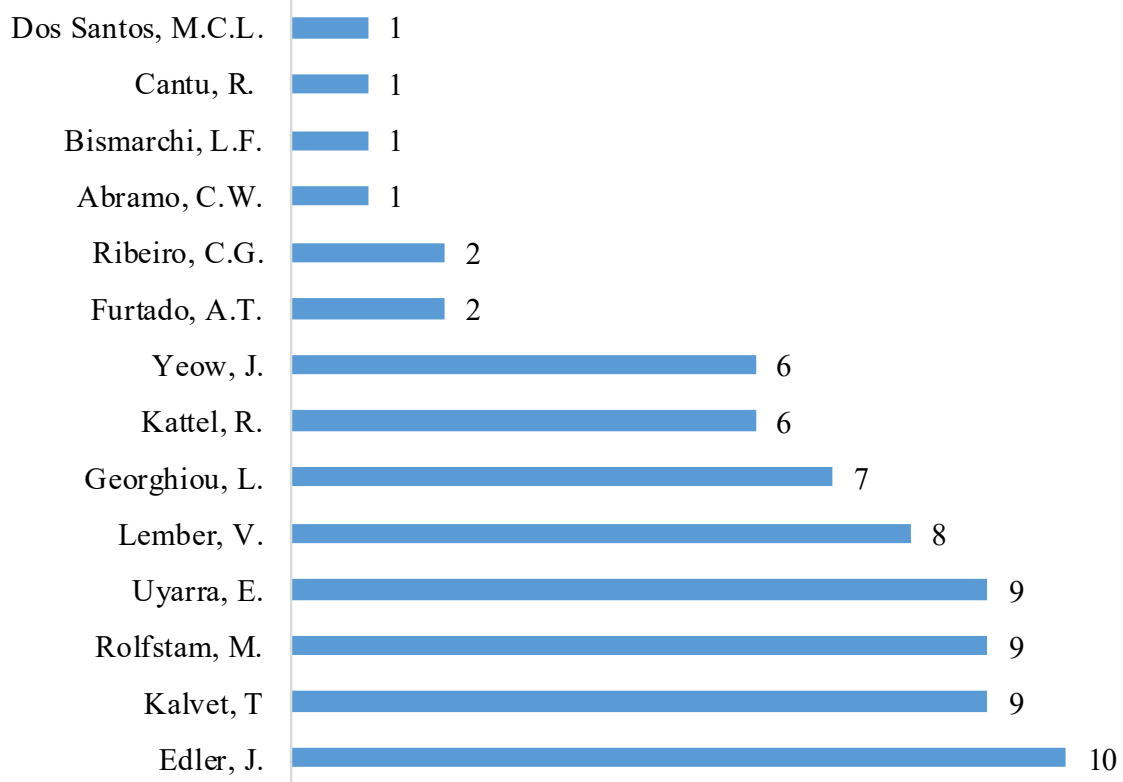

Fonte: Elaborada pelos autores deste artigo com dados da base Scopus (2018)

Com relação às universidades afiliadas (Figura 3) dos autores, tem-se em primeiro lugar a University of Manchester, com 18 publicações, em segundo a Manchester Business School com 11, a Aalborg Universitet com 10, a Tallinn University of Technology segue com nove, e somente após várias outras encontram-se as brasileiras Universidade Estadual Paulista e Universidade Estadual de Campinas, com 2 (duas), a Universidade Federal do Rio de Janeiro, a Universidade do Estado do Rio de Janeiro e a Universidade de São Paulo com 1 (uma) cada.

Entre as cinco universidades brasileiras, todas são públicas (federais ou estaduais), sendo três delas mantidas pelo Governo do Estado de São Paulo, justamente da região que mais investe em tecnologia e inovação no Brasil.

Figura 3 - Publicações por universidades

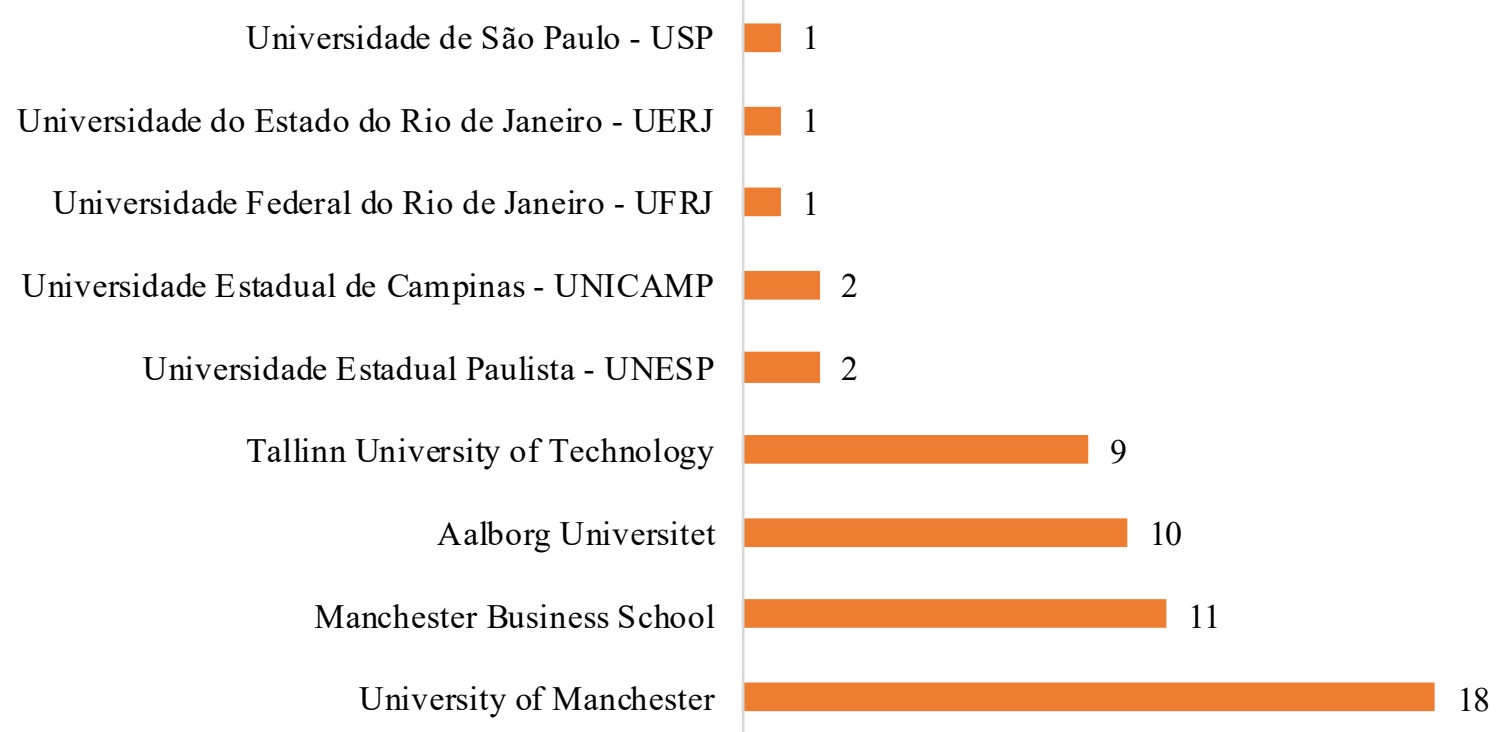

Fonte: Elaborada pelos autores deste artigo com dados da base Scopus (2018) 
Pela análise geográfica mundial, percebeu-se que o país com maior número de publicações é o Reino Unido, com um total de 42 publicações, exatamente o dobro de número de publicações da Itália que se encontra na segunda colocação no ranking. A Espanha e a Suécia dividem a terceira posição, ambas com 18 títulos cada. O Brasil aparece na $18^{a}$ posição, com cinco publicações ( $1^{\mathrm{a}}$ coluna da Tabela 2 ). Ainda é possível observar que o tema é mais abordado em países com maior grau de desenvolvimento industrial. Por certo, países mais desenvolvidos compreendem melhor a importância da inovação como fator de desenvolvimento econômico e social.

É importante analisar a quantidade de publicações desses países também ao longo do tempo, assim, demonstrou-se nas demais colunas da Tabela 2 suas publicações no período de 2005 a 2017.

Verifica-se na Tabela 2 que o Reino Unido apresenta uma distribuição mais uniforme de suas publicações durante os anos, já a Itália aumentou significativamente o número de suas publicações no último ano analisado (2017). O Brasil, a Suécia, a Dinamarca e a Estônia publicaram mais sobre o tema no ano de 2014, seguindo a tendência mundial que já foi apontada na Figura 1.

Tabela 2 - Publicações por área países no tempo

\section{Países}

United

Kingdom

$$
\text { Italy }
$$

Spain

Sweden

Denmark

Netherlands

United States

Estonia

China

Finland

France

Germany

Australia

Belgium

Croatia

Greece

Russian Fed.

Brazil

Total por ano
Total 2017201620152014201320122011201020092008200720062005

$\begin{array}{llllllllllllll}42 & 5 & 2 & 4 & 8 & 5 & 4 & 3 & 2 & 0 & 4 & 1 & 1 & 0\end{array}$

21

18

18

1700

$\begin{array}{lllll}14 & 1 & 2 & 2 & 0\end{array}$

$\begin{array}{lll}\mathbf{1 4} & 1 & 2 \\ \mathbf{1 1} & 1 & 0\end{array}$

$9 \quad 0 \quad 0$

12

15

80

0

3

82

1

$\begin{array}{ll}0 & 3 \\ 3 & 1\end{array}$

10

0

$\begin{array}{llllll}1 & 1 & 2 & 0 & 0 & 0\end{array}$

$$
8
$$

0

1

0

2

$\begin{array}{lll}1 & 1 & 1 \\ 2 & 2 & 0\end{array}$

0

0

0

3

$$
8
$$

$\begin{array}{ll}2 & 1 \\ 0 & 0\end{array}$

3
1

3

1
0

1

1

$\begin{array}{ll}0 & 1\end{array}$

0

$$
2
$$

$\begin{array}{ll}0 & 1\end{array}$

0

$\begin{array}{lll}1 & 1\end{array}$

$\begin{array}{llllll}0 & 0 & 1 & 0 & 0 & 2\end{array}$

8

7

$\begin{array}{ll}2 & 0 \\ 0 & 0\end{array}$

0

$\begin{array}{lll}1 & 0 & 0\end{array}$

.

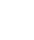


O tipo de documento predominante é o artigo, com 126 documentos, seguido de capítulo de livro, com 41, e papéis de conferências, com 35. Na Tabela 3 estão apresentadas as publicações por tipo de documento.

Tabela 3 - Publicações por tipo de documento

\begin{tabular}{ccc} 
Tipo de documento & Publicações & Percentual \\
Article & 126 & $53,6 \%$ \\
Book Chapter & 41 & $17,4 \%$ \\
Conference Paper & 35 & $14,9 \%$ \\
Review & 14 & $6 \%$ \\
Book & 8 & $3,4 \%$ \\
Conference Review & 4 & $1,7 \%$ \\
Article in Press & 3 & $1,3 \%$ \\
Editorial & 2 & $0,9 \%$ \\
Short Survey & 2 & $0,9 \%$ \\
Total & $\mathbf{2 3 5}$ & $\mathbf{1 0 0 \%}$ \\
\hline
\end{tabular}

Fonte: Elaborada pelos autores deste artigo com dados da base Scopus (2018)

O fato de terem sido localizados mais artigos de revista do que de papéis de conferência é um indicador de que essa é uma área consolidada cientificamente. Também a existência de um periódico (Innovation) direcionado para essa matéria especificamente, demonstra que o interesse pelo assunto é real e concreto. Na Tabela 4 estão apresentadas as publicações por tipo de documento na última década.

Tabela 4 - Publicações por tipo de documento na última década

\begin{tabular}{cccccccccccc} 
Tipos De Documentos & $\mathbf{2 0 1 7}$ & $\mathbf{2 0 1 6}$ & $\mathbf{2 0 1 5}$ & $\mathbf{2 0 1 4}$ & $\mathbf{2 0 1 3}$ & $\mathbf{2 0 1 2}$ & $\mathbf{2 0 1 1}$ & $\mathbf{2 0 1 0}$ & $\mathbf{2 0 0 9}$ & $\mathbf{2 0 0 8}$ & $\mathbf{2 0 0 7}$ \\
Article & 24 & 16 & 17 & 18 & 12 & 10 & 5 & 6 & 4 & 3 & 1 \\
Book Chapter & 2 & 1 & 3 & 20 & 2 & 1 & 8 & 1 & 1 & 0 & 0 \\
Conference Paper & 7 & 5 & 4 & 4 & 1 & 3 & 2 & 3 & 0 & 4 & 1 \\
Review & 3 & 1 & 0 & 1 & 0 & 2 & 0 & 0 & 0 & 1 & 1 \\
Book & 0 & 0 & 1 & 2 & 3 & 1 & 1 & 0 & 0 & 0 & 0 \\
Conference Review & 0 & 1 & 1 & 1 & 0 & 0 & 0 & 0 & 0 & 0 & 0 \\
Article in Press & 3 & 0 & 0 & 0 & 0 & 0 & 0 & 0 & 0 & 0 & 0 \\
Editorial & 0 & 0 & 1 & 0 & 0 & 0 & 1 & 0 & 0 & 0 & 0 \\
Short Survey & 0 & 0 & 0 & 0 & 0 & 0 & 0 & 2 & 0 & 0 & 0 \\
\hline
\end{tabular}

Fonte: Elaborada pelos autores deste artigo com dados da base Scopus (2018) 
Se considerados os tipos de documentos produzidos a cada ano na última década, é possível constatar que o número de artigos vem crescendo esporadicamente, tendo dobrado o número de publicações entre os anos 2011 e 2012 e realizado um saldo de 2016 para 2017. O número de capítulos de livro teve um aumento significativo no ano de 2014 comparado a todos os demais anos, apontando novamente o ápice de publicações relacionados ao tema nesse ano.

Ordenados os 235 documentos pela ordem de mais citados na última década, excluídas as autocitações, foram então selecionados os 40 primeiros colocados para uma melhor análise.

Por essa nova pesquisa, foi possível confirmar que o autor Edler possui domínio nesse tema. Além de ser o autor da obra mais citada 257 vezes na última década (Public procurement and innovation-Resurrecting the demand side), ele também possui mais outras quatro publicações, em parceria com outros autores, entre as 40 selecionadas, quais sejam: Policy instruments for public procurement of innovation: choice, design and assessment; Barriers to innovation through public procurement: a supplier perspective; Connecting demand and supply: the role of intermediation in public procurement of innovation; e Public procurement for innovation. Verificou-se que o fator de impacto da sua obra melhor referenciada (7.89) somente perdeu para a publicação dos autores Ang, G., Groosman, e M., Scholten, N. P. M., Dutch performance-based approach to building regulations and public procurement, com 10.27, e que se encontra na $21^{\text {a }}$ colocação.

O documento mais citado é um artigo e foi publicado no ano de 2007, entretanto, analisando a data dos demais, foi possível concluir mais uma vez o maior número de publicações da temática no ano de 2014. É importante esclarecer que não houve limitação sobre a data de publicação das obras, portanto, a seleção contém obras inclusive de 1984 e que continuam a ser referência para a temática. Obteve-se como resultado: ano 1984 (2 citações); 1993 (1); 1995 (1); 2005 (1); 2006 (2); 2007 (1); 2008 (3); 2009 (3); 2010 (3); 2011 (5); 2012 (5); 2013 (4); 2014 (8); e 2016 (1).

Destaca-se a predominância de publicações no periódico Research Policy, com sete artigos, sendo eles os $1^{\circ}, 3^{\circ}, 4^{\circ}, 5^{\circ}, 12^{\circ}, 27^{\circ}$ e $31^{\circ}$ colocados. Eles também possuem um bom fator de impacto: $7.89 ; 7.75 ; 5.48 ; 2.22 ; 1.24 ; 7.28 ;$ e 2.06 , respectivamente, sendo que a média de impacto das 40 publicações é de 3,54.

Todas as publicações analisadas são estrangeiras, não foi localizado nenhum documento brasileiro bem referenciado sobre essa temática.

Considerando as palavras-chave informadas pelos autores dos artigos selecionados, foi possível identificar no tema pesquisado a existência de outros subtemas, relacionados a políticas governamentais orientadas a incentivar a inovação e/ou a sustentabilidade, bem como redução dos impactos ambientais; parcerias público-privadas de cooperação; incentivos fiscais e econômicos para redução de custos nos projetos de inovação, entre outros. Era de se esperar que as palavras inovação e compras públicas aparecessem por diversas vezes, assim, a palavra innovation foi vista 30 vezes, sozinha ou acompanhada, a palavra public procurement foi localizada 20 vezes e purchasing apenas uma vez. Algumas palavras e suas derivações, associadas a outros termos, chamaram atenção pela quantidade de repetição: policy (15); demand (5); public (25); private/privatization (7); sustainable/sustainability (7); eco/clean/green/environmental impacts (5); R\&D/research/development/supplier survey (7); system/design (6); tendering/auctions (3); economy/regulation/cost/incentive (8); governance (2); risk management (2); strategic/decision-making/hurdle analysis/performance (5). 
Em uma revisão de literatura sobre o tema, a exclusão das 40 publicações ora analisadas da referência seria um grande lapso, pois essas obras constituem a base da pesquisa científica sobre Compras Públicas em Projetos de Inovação, no período de 2007 a 2017.

\section{Considerações Finais}

O objetivo proposto neste estudo foi realizar uma análise bibliométrica quantitativa acerca das Compras Públicas e Projetos de Inovação. O estudo bibliométrico forneceu alguns insights sobre as publicações existentes sobre o tema.

O levantamento das 235 publicações sobre essa temática selecionadas na base de dados internacional Scopus evidenciou os autores, as universidades, os países, os tipos de documentos utilizados para divulgação das pesquisas, as áreas a que o tema está vinculado, e os periódicos mais alinhados com o tema ao longo do tempo até 2017. Os resultados oriundos da ordenação dos documentos por sequência de número de citações na última década, por sua vez, permitiram construir um portfólio bibliográfico composto de 40 publicações mais relevantes.

Após a realização de análise bibliométrica sobre os resultados, constatou-se que o campo do conhecimento pesquisado apresentou uma grande curvatura ascendente em 2014 em termos de volume de publicações internacionais, principalmente de artigos, e que o número de publicações brasileiras ainda é muito baixo, havendo uma clara lacuna na literatura brasileira sobre o tema e a necessidade de maior debate acerca da área, tendo em vista que a inovação é uma tendência global.

Em relação à interdisciplinaridade do tema em questão, observa-se que ele vem sendo tratado por diversas áreas do conhecimento, por exemplo: negócios, gestão e contabilidade; ciências sociais; engenharia; economia, econometria e finanças; ciência ambiental; ciência da computação; entre outras.

Os resultados destacaram cinco universidades brasileiras que publicaram na área, todas públicas (federais ou estaduais) e três delas paulistas. Denota-se disso que o posicionamento do Brasil nas pesquisas sobre esse assunto ainda é de iniciante e não é surpreendente o fato de que a maior parte das pesquisas foram realizadas em São Paulo, que é o Estado que mais investe em tecnologia e inovação no país.

Conforme explanado no início do presente artigo, a inovação tecnológica, que impulsiona o desenvolvimento econômico do país, possui características de alta complexidade e demanda que empresas assumam grandes riscos para se manterem em destaque no mercado, necessitando gerar novas ideias e impulsionar projetos de inovação cada vez mais dinâmicos e ágeis.

A compra de materiais e de produtos já foi identificada como o maior risco dentro dos projetos de inovação, que, muitas vezes, não consideram de forma adequada a importância da gestão do processo de aquisições. Os projetos de inovação em execução demandam materiais e produtos específicos que possuem características bastante peculiares e que precisam estar disponíveis no momento exato em que se fazem necessários, de acordo com o estipulado no plano de trabalho aprovado.

Mesmo assim, como se notou no estudo ora apresentado, não foram encontradas muitas publicações sobre planejamento e controle das compras em projetos de inovação, principalmente no Brasil. 
A gestão de aquisições é um desafio para as empresas atuantes no desenvolvimento de novos produtos e/ou tecnologias. E, apesar da relevância dos processos de compras para a boa execução dos projetos de inovação, não existem ainda bons estudos e normativos que forneçam conteúdos suficientes para proporcionar às instituições nacionais informações sobre as formas adequadas de gerir a aquisição de itens ou de equipamentos imprescindíveis para seus projetos, o que é agravado, em grande medida, no ambiente público.

Por fim, o tema ainda demanda muitos estudos com potencial de melhorar a atual condução dos projetos de inovação idealizados e executados no Brasil e contribuir, portanto, com o desenvolvimento tecnológico, econômico e social do nosso país.

\section{Perspectivas Futuras}

Cumpre salientar, concluindo, que o estudo sobre essa temática no Brasil é ainda muito raso, por isso há enorme potencial para seu desenvolvimento. Um futuro trabalho poderá analisar o portfólio bibliográfico internacional levantado sob o aspecto qualitativo, com a finalidade de se criar um referencial teórico sobre essa temática, com aprofundamento nas obras mais referenciadas e interpretação das teorias apresentadas por cada autor adequando-as à realidade brasileira.

Ressalta-se que não foi localizado nenhum documento brasileiro bem referenciado sobre essa temática. Assim, ainda há muito campo de estudo em nosso país, considerando em especial nossas peculiaridades no sistema de compras públicas.

A preocupação com as compras governamentais vem aumentando. Nota-se que a legislação brasileira está procurando se adequar aos novos tempos (é o caso da nova Lei de Licitações: PL n. 4.253/2020) e que mais pesquisas e estudos na temática Compras Públicas em Projetos de Inovação serão necessários para que tenhamos maior chances de competir no mercado internacional com produtos mais tecnológicos e inovadores.

\section{Referências}

ARAÚJO, C. A. Bibliometria: evolução histórica e questões atuais. Em Questão, Porto Alegre, v. 12, n. 1, p. 11-32, 2006.

BARBALHO, S.; RICHTER, E. H.; ROZENFELD, H. Melhorando o processo de aquisição de materiais e componentes para protótipos de novos produtos. Gestão Industrial, [s.l.], v. 4, n. 3, p. 22-33, 2008.

BARKI, H.; RIVARDI, S.; TALBOT, J. A keyword classification scheme for IS research literature: an update. Management Information Systems Quarterly, [s.l.], 17, n. 2, p. 209-226, 1993.

BOUYSSOU, D.; MARCHANT, T. Ranking scientists and departments in a consistent manner.

Journal of the American Society for Information Science and Technology, [s.l.], v. 62, n. 9, p. 1.761-1.769, 2011.

CORRÊA, H. L.; CORRÊA, C. A. Administração da produção e operações: manufatura e serviços, uma abordagem estratégica. 3. ed. São Paulo: Atlas, 2012. 
DAIM, T. U. et al. Forecasting emerging Technologies: use of bibliometric and patentee analysis. Technological Forecasting and Social Change, [s.l.], v. 73, p. 981-1.012, 2006.

DE BELLIS, N. Bibliometric and citation analysis: from the science citation index to cyber metrics. Lanham: The Scarecrow Press, 2009.

ELAM, J.; HUBER, G.; HURT, M. An examination of the DDS Literature (1975-1985). In: MCLEAN, E. R.; SOL, H. (ed.). Decision support systems: a decade in perspective. Amsterdam: Elsevier Science, 1986.

FRANCK, G. Scientific communication: a vanity fair? Science, [s.l.], v. 286, n. 5.437, p. 53-55, 1999.

FRIPONG, Y.; OLUWOYE, J.; CRAWFORD, L. Causes of delay and cost overruns in construction of groundwater projects in developing countries; Ghana a case study. International Journal of Project Management, [s.l.], v. 21, p. 321-326, 2003.

GUIA PMBOK. Um Guia do Conhecimento em Gerenciamento de Projetos. 5. ed. São Paulo: Global Standard, 2013. p. 355-389.

GOELZER, V. et al. Análise de um Processo de Inovação a partir da Ótica de Gestão de Projetos.

Gestão e Projetos, [s.l.], v. 5, n. 2, p. 78-89, 2014.

GROOS, O. V.; PRITCHARD, A. Documentation notes. The Journal of Documentation, v. 25, n. 4, p. 344-349, 1969.

MANAVAZHI, M. R.; ADHIKARI, D. K. Material and equipment procurement delays in highway projects in Nepal. International Journal of Project Management, [s.l.], v. 20, p. 627-632, 2002.

MEHO, L. I. The rise and rise of citation analysis. Physics World, [s.l.], v. 20, n. 1, p. 32-36, 2007.

MERIGÓ, J. M.; GIL-LAFUENTE, A. M.; YQGER, R. R. An overview of fuzzy research with bibliometric indicators. Applied Soft Computing, [s.l.], v. 27, p. 420-433, 2015.

PRITCHARD, A. Statistical bibliography or bibliometric? Journal of Documentation, [s.l.], v. 25, n. 4, p. 348-349, 1969.

REIS, A. C. B.; BARBALHO, S. C. M.; ZANETTE, A. C. D. A bibliometric and classification study of project-based learning in engineering education. Production, [s.l.], 2017.

SALTON, G.; MCGILL, M. J. Introduction to modern information retrieval. New York: Mc-Graw Hill, 1983.

SCOPUS. [Base de dados - Internet]. [2018]. Disponível em: https://www.scopus.com/home.uri. Acesso em: 25 de jun. 2018.

TAPPING, D.; SHUKER, T. Value stream management for the lean office: eight steps to planning, mapping and sustaining lean improvements in administrative areas. Productivity Press, New York, 2003.

ZHAO, D.; LOGAN, E. Citation analysis using scientific citations on the Web as a data source: a case study in the XML research area. Scientometrics, [s.l.], 54, n. 3, p. 449-472, 2002. 


\section{Sobre os Autores}

\section{Luciana Maria de Oliveira Cortinhas}

E-mail: lucianamaria@unb.br

ORCID: https://orcid.org/0000-0001-7615-1575

Mestre em Propriedade Intelectual e Transferência de Tecnologia para a Inovação pela Universidade de Brasília em 2019.

Endereço profissional: Campus Universitário Darcy Ribeiro, Auditoria Interna, Bloco Eudoro de Souza (BAES), $1^{\circ}$ andar, sala A1-57/11, Brasília, DF. CEP: 70904-109.

\section{Sanderson César Macedo Barbalho}

E-mail: sandersoncesar@unb.br ORCID: https://orcid.org/0000-0003-1664-4866

Doutor em Engenharia Mecânica pela Universidade de São Paulo em 2006.

Endereço profissional: Campus Universitário Darcy Ribeiro, Faculdade de Tecnologia, Departamento de Engenharia da Produção, Brasília, DF. CEP: 70910-900. 